\title{
26 Research Square \\ U19-Net: A Deep Learning Approach for Obstacle Detection in Self-Driving Cars
}

\author{
Albert Aarón Cervera-Uribe ( $\nabla$ albert.cervera.edu@gmail.com ) \\ Universidad Autónoma de Yucatán: Universidad Autonoma de Yucatan https://orcid.org/0000-0002- \\ 4000-6250
}

\section{Paul Erick Mendez-Monroy}

Universidad Nacional Autónoma de México: Universidad Nacional Autonoma de Mexico

\section{Research Article}

Keywords: Deep learning, Self-driving cars, Neural networks, Encoder-Decoder, Detection

Posted Date: June 28th, 2021

DOl: https://doi.org/10.21203/rs.3.rs-520174/v1

License: (1) This work is licensed under a Creative Commons Attribution 4.0 International License. Read Full License

Version of Record: A version of this preprint was published at Soft Computing on April 7th, 2022. See the published version at https://doi.org/10.1007/s00500-022-06980-6. 


\section{Abstract}

Development of self-driving cars aims to drive safely from one point to another in a coordinated system where the on-board autonomous vehicle system should react and possibly alert drivers about the driving environments and possible collisions that may arise between drivers and obstacles. In order to achieve a high level of autonomy in urban scenarios with unpredictable traffic, these systems must have robust and reliable obstacles detection systems. This work proposes U19-Net, a deep learning model that explores improvement of the so-called encoder-decoder neural networks with a very deep network architecture. In particular, U19-Net is applied and successfully evaluated for the vehicle and pedestrian detection tasks within an open source dataset consisting of frames from a video in real driving scenarios. The output of the network consists of a pixel-level mask that identifies each pixel as vehicle or pedestrian, demonstrating that the depth representation attained with U19-Net is beneficial in this kind of architecture for vision systems in self-driving cars.

\section{Full Text}

This preprint is available for download as a PDF. 